\title{
Competitividad y desempeño económico del sector silvoagropecuario a nivel regional en Chile
}

\author{
Competitiveness and economic performance of the agrosilvo-pastoral \\ sector at a regional level in Chile
}

Luz María Ferrada ${ }^{1 *}$, Johanna Candia², Claudia Pérez ${ }^{3}$

\section{RESUMEN}

El sector silvoagropecuario aporta de manera significativa tanto al PIB como al empleo regional en el sur de Chile. El objetivo de la investigación es analizar este sector en cuanto a su competitividad y resultados económicos en las regiones de O'Higgins, Maule, La Araucanía y Los Lagos. La competitividad se estudia a partir de un análisis multivariado que incluye cinco factores: capital humano, personas y trabajo, infraestructura comunitaria, rendimiento de recursos y desempeño económico, estimados mediante indicadores sintéticos; luego se evalúa el impacto de cada factor sobre los resultados económicos. Entre otros se obtiene que la dotación de factores difiere por territorio y el factor más relevante es la disponibilidad de capital humano, explicado de preferencia por las variables cobertura preescolar y matriculada en cursos de pre y postgrados. Además, este y personas y trabajo impactan de modo sinificativo sobre los resultados económicos del sector en el territorio.

Palabras clave: economía silvoagropecuaria, competitividad, economía regional.

\begin{abstract}
The agrosilvo-pastoral sector contributes significantly both to the GDP and to regional employment levels in southern Chile. The aim of this study is to analyze the agrosilvo-pastoral sector in terms of its competitiveness and economic results in the regions of O'Higgins, Maule, Araucanía and Los Lagos. Competitiveness is studied by undertaking a multivariate analysis that includes five factors: human capital, people and work, community infrastructure, resource management efficiency and economic performance, in order to create synthetic indicators. Subsequently, the impact of different factors on the economic results is analysed. One of the results obtained indicates that endowment of these factors varies spatially and that the factor with the greatest impact is availability of human capital, explained, mainly, by the variables pre-school coverage and matriculation in pre and postgraduate courses. Furthermore, the effect of the factors human capital and people and work factor on economic results significative in this sector is significant.
\end{abstract}

Key words: agrosilvo-pastoral economy, competitiveness, regional economy.

\section{Introducción}

Las regiones en Chile se especializan de preferencia en sectores extractivos. De esta forma, el sector silvoagropecuario es fundamental en la economía de las regiones de O'Higgins, Maule, La Araucanía, Los Ríos y Los Lagos. Este sector demanda entre 30\% (en el Maule) y 18\% (en Los Lagos) del empleo regional (INE, 2009 a 2011) y aporta al PIB regional más de tres veces de lo que el sector lo hace al país (Banco Central, 2009 a 2011). Sin embargo, el desempeño económico de estas regiones suele ser más bajo que en otras. Un argumento que podría explicar este resultado, siguiendo la lógica de la teoría de las etapas de crecimiento de las regiones (Cuadrado, 1992: 528), es la relación inversa entre desarrollo económico e importancia relativa de los sectores primarios en el PIB, así se esperarían mejores condiciones en la medida que la función de producción regional se traslade a sectores productivos con mayor valor agregado. Otra explicación es que el nivel de competitividad sectorial pudiese estar aún bajo un umbral deseable, este es el enfoque con que se aborda el tema en este trabajo.

\footnotetext{
1 Universidad de Los Lagos, Núcleo de Economía Regional, Departamento de Ciencias Administrativas y Económicas, Avda. Fuchslocher 1305, Osorno, Chile.

2 Vicerrectoría de Finanzas, Universidad de Los Lagos.

3 Unidad de Transferencia Tecnológica, Universidad de Los Lagos.

* Autor para correspondencia: lferrada@ulagos.cl
}

Fecha de Recepción: 27 Mayo, 2014.

Fecha de Aceptación: 22 Septiembre, 2014. 
El objetivo de esta investigación es analizar el sector silvoagropecuario en cuanto a su competitividad y resultados económicos desde una perspectiva regional, para verificar la existencia de diferencias de competitividad sectorial entre regiones. Así se tienen los siguientes objetivos específicos: (1) analizar la competitividad sectorial en las regiones que se especializan de forma notable en el sector silvoagropecuario y (2) evaluar el impacto de distintos factores no económicos de la competitividad sectorial en el desempeño económico del sector en las regiones.

Aquí conviene detenerse en el concepto de competitividad y su relación con lo sectorial y regional.

La literatura plantea que desde un enfoque territorial el concepto de competitividad puede variar según el objeto de estudio, ya sea nacional, supranacional o subnacional; el Foro Económico Mundial (FEM) entiende que un territorio es competitivo en cuanto a que "el conjunto de instituciones, políticas y factores determinen un nivel de productividad" (Schwab, 2011: 3-4). Así, el Centro Mundial de Competitividad (WCC) estima un indicador desde 1989 a base de las dimensiones: desempeño económico, eficacia del gobierno, eficiencia en los negocios e infraestructura, cada una compuesta por cinco factores y ellos por variables.

No obstante, desde una perspectiva global, Hatzichronoglou (1996: 20) considera que la competitividad es "la capacidad de las empresas, industrias, regiones, naciones o áreas supranacionales para generar -a la vez que se ven expuestas a la competencia internacional- niveles altos de ingreso y empleo de forma sostenible". Esta lógica permite acercarse a un concepto sectorial. Marcovitch (1994: 4) plantea dicha orientación indicando que la competitividad es "la capacidad de los sectores económicos de generar bases de creación y desarrollo de ventajas que sustenten una posición competitiva internacional, y sectorialmente en la medida en que un sector económico ofrezca potencial para crecimiento y retornos sobre las inversiones atractivas para las empresas que lo componen".

Ahora bien, Porter (1995: 71-78) desarrolla la idea de competitividad como "un proceso altamente localizado que promueve la especialización y la eficacia local; así, cada lugar ofrece ventajas específicas dependiendo de sus diversos factores de atracción, las ventajas competitivas". A su vez,
Araoz (1997: 6) indica que la competitividad es "el resultado del entretejido de una serie de factores económicos, geográficos, sociales y políticos que conforman la base estructural del desarrollo de una nación".

Considerando las definiciones, en este trabajo se entenderá por competitividad sectorial a la capacidad de un sector de mantener una posición competitiva en términos espaciales, mediante una oferta sostenible de bienes y servicios, tanto al mercado interno como externo, que le permita generar crecimiento, empleo y bienestar social, en virtud de la inversión realizada tanto en capital humano como en infraestructura, propiedad privada y común. De esta forma es un proceso localizado espacialmente, dinámico y de largo plazo.

En adelante el artículo se estructura de la siguiente forma: primero se describe la metodología y los datos a utilizar, para presentar los resultados y concluir.

\section{Materiales y Métodos}

El territorio a analizar lo componen las regiones de O'Higgins, Maule, La Araucanía, Los Ríos y Los Lagos (Figura 1). Todas ellas se especializan marcadamente en el sector durante el período de

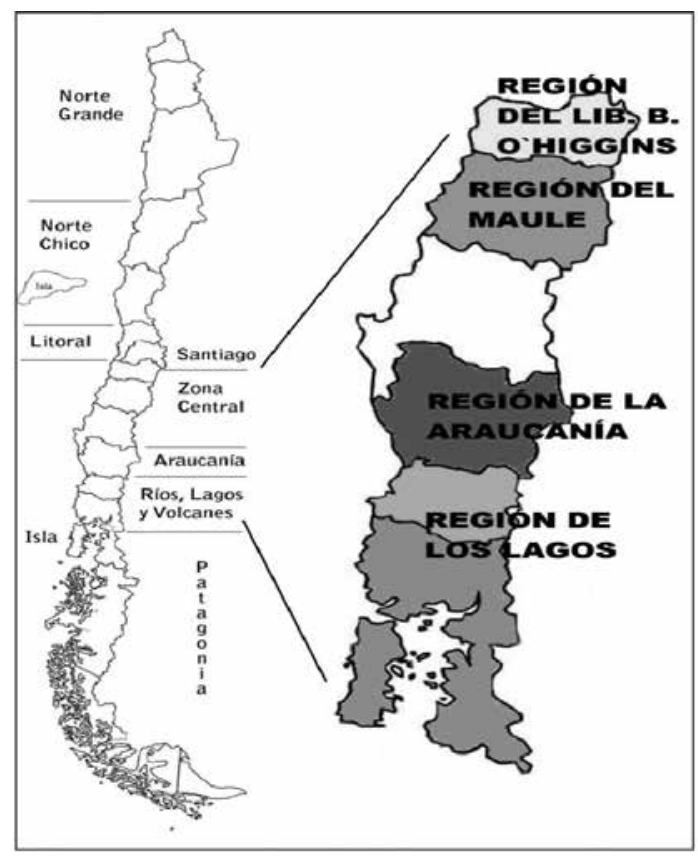

Figura 1. Regiones estudiadas. 
análisis, esto es entre los años 2000 y 2011, lo que corresponde a un período adecuado, que permite contar con información comparable en las variables de interés, para cumplir con los propósitos del trabajo. Por lo mismo, se considera la división político administrativa de Chile hasta el 2007, es decir, que la región de Los Lagos incluye a la actual región de Los Ríos.

En este estudio se estima un índice de competitividad en términos relativos, es decir, comparando las regiones mencionadas en el ámbito del sector silvoagropecuario dado los antecedentes del período 2000 a 2011, evaluando el impacto de los factores explicativos de la competitividad en el crecimiento económico sectorial por región.

\section{Construcción del índice de competitividad regional}

Basados en la definición de competitividad indicada en el punto anterior, la propuesta de factores del Centro Mundial de Competitividad, las particularidades del sector económico a estudiar y la disponibilidad de variables, se definen en cinco dimensiones o factores indicativos de competitividad: economía (EC), capital humano $(\mathrm{CH})$, personas y trabajo (PT), infraestructura comunitaria (IC) y rendimiento de recursos (RR). Cada factor se mide a partir de un índice sintético anual, procediendo de la siguiente forma:

$1^{\circ}$. Se seleccionan variables indicativas de cada uno de los factores mencionados.

$2^{\circ}$. Se clasifican las variables entre aquellas que afectan positiva y negativamente al factor de competitividad.

$3^{\circ}$. Cada variable se estandariza según se indica:

- Para las que afectan positivamente a la competitividad, Z se obtiene como:

Zirt $=\frac{X_{i r}-\operatorname{mínimo}\left(X_{i r}, \forall r, t ; r=1, \ldots R \text { y } t=1, \ldots T\right)}{\operatorname{máximo}\left(X_{i n}, \forall r, t ; r=1, \ldots R \text { y } t=1, \ldots T\right)-\text { mínimo }\left(X_{i n}, \forall r, t ; r=1, \ldots R \text { y } t=1, \ldots T\right)}$

- Para las variables que afectan negativamente a la competitividad, $\mathrm{Z}$ se obtiene como:

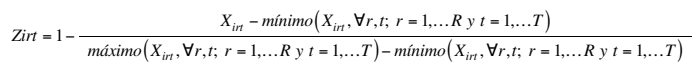

Siendo $Z_{i r t}$ la variable estandarizada entre el conjunto de datos para la variable $i$, para $\mathrm{T}$ años en $\mathrm{R}$ regiones. En este caso $\mathrm{T}=12$ años (2000 a 2011) y $\mathrm{R}=4$ regiones.

$4^{\circ}$. Se obtiene un índice de competitividad parcial $\left(\mathrm{ICP}_{\mathrm{ir}}\right)$ por año, región y en cada factor, ponderando cada variable de la siguiente forma:

$$
I C P_{r t}=\sum_{i=1}^{N} Z_{i r t} * P_{i}
$$

Donde $\mathrm{N}$ es el número de variables por dimensión, $P_{i}$ es el peso de la variable $i$, que se obtiene de una proporción entre la desviación estándar de la variable para todos los años entre las regiones:

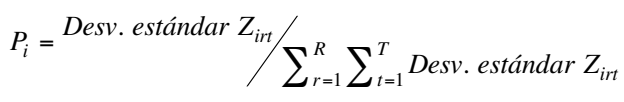

Así, cada variable tiene un único $P_{i}$.

$5^{\circ}$. El Índice de Competitividad Parcial por dimensión en el conjunto de años en cada región se estima como un promedio:

$$
I C P_{r, f}=\frac{\sum_{t=1}^{T} I P_{r t}}{12}
$$

Por lo tanto, cada indicador se obtiene en términos relativos en una escala de 0 a 1 .

$6^{\circ}$. El Índice de Competitividad Global por región para el total de las dimensiones $\left(I C G_{r}\right)$ se estima como:

$$
I C G_{r}=\frac{\sum_{f=1}^{5} I C P_{r f}}{5}
$$

\section{Evaluación impacto de factores sobre resultados económicos}

En este caso se trata de evaluar cómo impactan los distintos ámbitos de la competitividad en el desempeño económico, es decir, en cada una de las variables que componen el factor economía, luego se calcula el efecto de las regiones sobre las mismas variables; para ello se plantean cuatro ecuaciones, una por variable dependiente (PIB percápita, $P I B$; exportaciones del subsector agrícola, XAGRI; exportaciones del subsector pecuario, XPECUA, exportaciones del subsector forestal, XFOREST, e inversiones FIA), para luego repetir el proceso pero incorporando a las regiones. 
Cada uno de los cuatro modelos se estima con 48 unidades muestrales, esto es 12 períodos para las 4 regiones, como un panel, de la siguiente forma:

$$
Y_{r t}=\beta_{0}+\beta_{1} C T_{r t}+\beta_{2} P T_{r t}+\beta_{3} I C_{r t}+\beta_{4} R R_{r t}+\mu_{r t}
$$

Donde $Y$ puede ser PIB, XAGRI, XPECUA, XFOREST y FIA indicando a las variables dependientes de cada ecuación, cada una ingresa al modelo en forma estandarizadas. Luego, las variables dependientes son factores indicativos de la competitividad (sin incluir EC cuyas variables estan en la dependiente), los que han sido estimados como índices sintéticos, por lo que se consideran exógenas.

Luego se incorporan las regiones, siendo la región de Los Lagos la referencia, así su impacto estaría incorporado en la constante.

$$
\begin{gathered}
Y_{r t}=\beta_{0}+\beta_{1} C T_{r t}+\beta_{2} P T_{r t}+\beta_{3} I C_{r t}+\beta_{4} R R_{r t}+\text { ARAUCANÍA }+ \\
+ \text { MAULE }+O^{\prime} H I G G I N S+\mu_{r t}
\end{gathered}
$$

Mediante análisis de significación estadística se verifica el impacto de todos los factores en cada una de las variables que se asocian a desempeño económico y se verifica si hay impacto de las regiones, es decir, si el impacto cambia entre los territoriales.

\section{Datos}

Se obtiene información de 40 variables, de diferentes bases de datos (Tabla 1). La disponibilidad para todo el período es diversa, por lo que se usan los siguientes criterios:

- Para los años en que no hay información se repite el dato del período anterior. Se procede así cuando se usa como base de datos la encuesta CASEN, en las variables inversión del FIA y consultorios urbanos y rurales.

- A partir de 2010 algunos datos regionales vienen separados, para Los Ríos y Los Lagos, en este caso se ponderó por la proporción de población en cada una.

- En el caso de datos atípicos se reemplaza por el valor de tendencia, ello ocurre en rendimiento de cebada para la región de O’Higgins en 2004 y 2010.
Las variables se calculan según población, PIB o superficie y se ordenan por factor (Tabla 1). Todas ingresan al indicador con signo positivo, con excepción de porcentaje de pobreza, denuncias por delitos y porcentaje de hogares con ingresos bajos, que se entiende afectan negativamente a la competitividad.

\section{Resultados y Discusión}

Como se ha indicado, se estima un índice de competitividad sectorial regional. Aquí se presentan los resultados, primero del índice global (ICG) y luego del parcial (ICP), para posteriormente explicarlos a partir de la evolución de los indicadores, y finalmente analizar el impacto de los factores en el desempeño económico sectorial regional.

\section{Índice de competitividad global}

La competitividad sectorial, para el conjunto de factores en el período, muestra diferencias importantes entre regiones, de manera que es posible apreciar un ranking (Figura 2), en el que O'Higgins obtiene una posición favorable en todo el período (Figura 3). No obstante, la posición relativa de las otras regiones varía en el tiempo, destacando el dinamismo de la región de Los Lagos con una evolución positiva.

\section{Índice de Competitividad Parcial}

Se obtiene que el factor economía es el que observa mayores diferencias entre regiones (Figura 4), siendo O'Higgins la con mayor indicador, con lo que evidencia la importancia que tiene el desempeño económico para el indicador global. Menos dispersión regional observan las dimensiones capital humano y personas y trabajo aunque resultan ser decisivas en el ranking global.

Llama la atención el bajo resultado que obtiene la región del Maule, a pesar sus altos indicadores de capital humano, infraestructura comunitaria y buen desempeño económico. La Araucanía obtiene una mejor posición relativa, pero en este caso está acompañado también de buenos resultados en rendimiento de recursos. El menor desempeño de la región de Los Lagos se asocia principalmente al factor economía.

Ahora bien, ¿cómo es la evolución de cada factor en el período de estudio? y ¿Qué aspectos 
Tabla 1. Variable según factor: desviación estándar (DE), valor mínimo (min), rango entre 2000 y 2011 y ponderación (pond.).

\begin{tabular}{|c|c|c|c|c|c|}
\hline Factor & Variable & D.E. & Mín. & Rango & Pond. \% \\
\hline \multirow{7}{*}{$\begin{array}{l}\text { Capital } \\
\text { humano }\end{array}$} & Promedio puntaje SIMCE & 0,226 & 238,00 & 26,500 & 12,7 \\
\hline & $\%$ de cobertura de educación prebásica neta & 0,253 & 18,087 & 27,404 & 14,3 \\
\hline & $\%$ de cobertura de educación básica neta & 0,314 & 92,764 & 2,747 & 17,7 \\
\hline & $\%$ de cobertura de educación media neta & 0,244 & 52,989 & 21,852 & 13,7 \\
\hline & Escolaridad promedio de población 15 años y más & 0,208 & 8,499 & 1,235 & 11,7 \\
\hline & $\%$ de matriculados pregrado sector agrícola & 0,289 & 8,045 & 10,862 & 16,3 \\
\hline & $\%$ de matriculados postgrado sector agrícola & 0,237 & 0,000 & 32,899 & 13,3 \\
\hline \multirow{10}{*}{$\begin{array}{c}\text { Personas } \\
\text { y } \\
\text { trabajo }\end{array}$} & Tasa bruta de natalidad & 0,222 & 12,967 & 3,473 & 9,6 \\
\hline & $\%$ población cotizante en FONASA & 0,275 & 30,539 & 58,592 & 11,9 \\
\hline & \% población cotizante en ISAPRE & 0,322 & 3,348 & 3,102 & 14,0 \\
\hline & $\%$ población mayor de 15 años en fuerza de Trabajo & 0,186 & 45,573 & 18,229 & 8,0 \\
\hline & $\%$ población mayor de 15 años ocupada sector & 0,255 & 7,343 & 10,377 & 11,0 \\
\hline & silvoagropecuario & & & & \\
\hline & $\begin{array}{l}\% \text { población mayor de } 15 \text { años en la fuerza de trabajo } \\
\text { capacitada ocupada dependiente }\end{array}$ & 0,227 & 16,887 & 26,872 & 9,8 \\
\hline & \% población en situación de pobreza & 0,269 & 9,958 & 21,587 & 11,7 \\
\hline & $\%$ de denuncias totales de delitos & 0,245 & 5,410 & 14,481 & 10,6 \\
\hline & Hogares en $40 \%$ menor de ingresos autónomos & 0,296 & 10,021 & 6,594 & 12,8 \\
\hline \multirow{9}{*}{$\begin{array}{l}\text { Rendimiento } \\
\text { de } \\
\text { recursos }\end{array}$} & $\%$ superficie forestada y reforestación & 0,227 & 0,123 & 1,229 & 9,6 \\
\hline & Rendimiento regional cultivos año trigo & 0,219 & 31,600 & 116,200 & 9,2 \\
\hline & Rendimiento regional cultivos año avena & 0,208 & 10,400 & 103,700 & 8,8 \\
\hline & Rendimiento regional cultivos año cebada & 0,218 & 10,500 & 110,100 & 9,2 \\
\hline & Rendimiento regional cultivos año papas & 0,216 & 125,20 & 670,500 & 9,1 \\
\hline & $\begin{array}{l}\% \text { superficie plantaciones forestales pino y eucalipto } \\
\text { industriales regionales }\end{array}$ & 0,335 & 0,120 & 14,603 & 9,1 \\
\hline & Cabeza de ganado bovino por hectárea & 0,323 & 0,007 & 0,036 & 13,6 \\
\hline & Cabeza de ganado ovino por hectárea & 0,270 & 0,000 & 0,008 & 11,4 \\
\hline & Cabeza de ganado porcino por hectárea & 0,345 & 0,004 & 2,523 & 14,6 \\
\hline \multirow{10}{*}{$\begin{array}{l}\text { Infraestructura } \\
\text { comunitaria }\end{array}$} & $\%$ hogares con sistema distribución agua urbano & 0,146 & 46,903 & 24,631 & 5,8 \\
\hline & $\%$ hogares con sistema distribución de agua rural & 0,169 & 21,409 & 11,862 & 6,8 \\
\hline & $\%$ hogares con conexiones a internet & 0,207 & 5,334 & 23,493 & 8,3 \\
\hline & $\begin{array}{l}\text { Establecimientos educacionales municipales rurales por } \\
1.000 \text { personas }\end{array}$ & 0,291 & 0,0008 & 0,001 & 11,7 \\
\hline & $\begin{array}{l}\text { Establecimientos educacionales municipales urbanos } \\
\text { por } 1.000 \text { personas }\end{array}$ & 0,258 & 0,0002 & 0,0001 & 10,4 \\
\hline & Laboratorios de salud municipal & 0,291 & 0,868 & 1,564 & 11,7 \\
\hline & Consultorio rural por persona población rural & 0,258 & 0,242 & 0,137 & 10,4 \\
\hline & Consultorio urbano por persona población urbana & 0,232 & 0,063 & 0,282 & 9,3 \\
\hline & Posta de salud rural por persona & 0,336 & 2,560 & 4,318 & 13,5 \\
\hline & SAPU por persona & 0,286 & 0,009 & 0,180 & 11,5 \\
\hline \multirow{5}{*}{ Economía } & Exportaciones agrícolas per cápita en dólares & 0,276 & 3,932 & 2.088 & 21,4 \\
\hline & Exportaciones pecuarias per cápita en dólares & 0,254 & 1,429 & 693 & 19,7 \\
\hline & Exportaciones forestales per cápita en dólares & 0,237 & 1,579 & 536 & 18,3 \\
\hline & PIB silvoagropecuario per cápita & 0,299 & 119,28 & 503 & 23,2 \\
\hline & Inversión FIA por cada 1.000 habitantes & 0,224 & 242,28 & 1.077 & 17,4 \\
\hline
\end{tabular}

Fuente: Elaboración propia. 


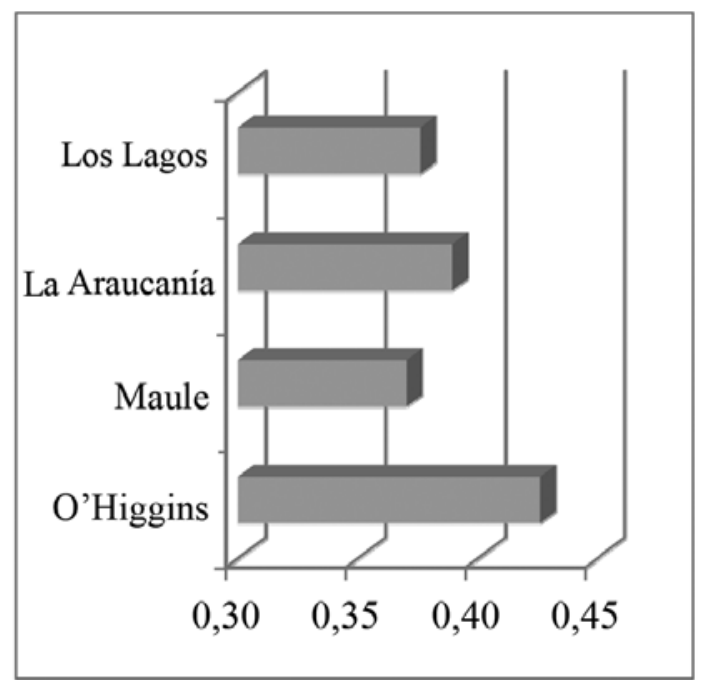

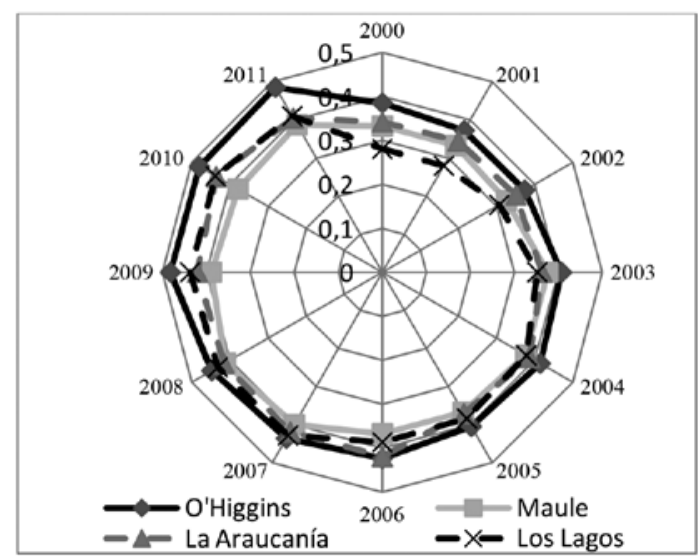

Figura 3. Índice de competitividad global por año y región. Fuente: Elaboración propia.

Figura 2. Índice de competitividad global por región.

Fuente: Elaboración propia.

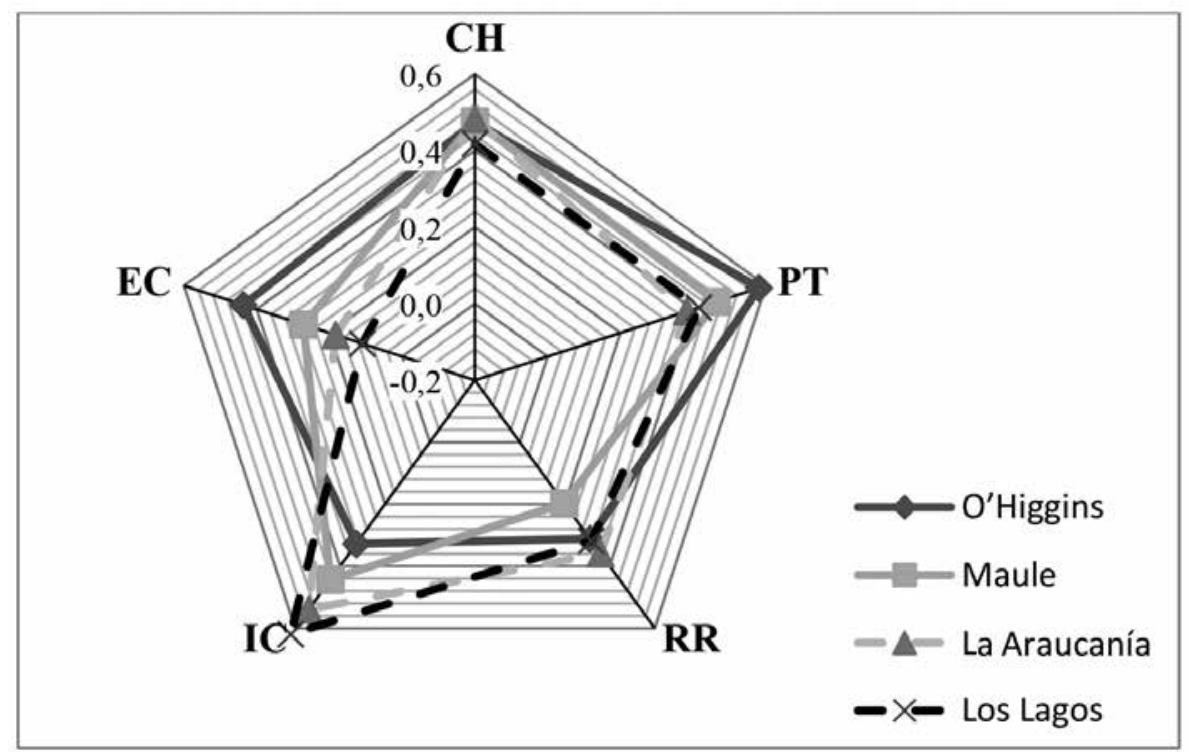

Figura 4. Índice de competitividad parcial por factor y región.

Fuente: Elaboración propia.

EC: Desempeño Económico; CH: Capital Humano; PT: Personas y Trabajo; RR: Rendimiento de Recursos; IC: Infraestructura Comunitaria. 
de la competitividad tienen mayor impacto en el desempeño económico sectorial de las regiones? Esto es lo que se analiza a continuación.

\section{Análisis de la Evolución por Factor}

\section{Desempeño Económico}

El desarrollo económico del sector tradicionalmente es analizado a partir del comportamiento del producto interno bruto sectorial per cápita, en este caso se estima un indicador que incluye al PIB y se agregan las variables inversión en la investigación del sector agropecuario (FIA) debido a la importancia que tiene el mercado externo en dichos rubros para el desempeño económico sectorial y las exportaciones de cada subsector relevante: agrícola, pecuario y forestal.

Pues bien, se calcula un indicador de desempeño económico para dichas regiones por año y se observa que la evolución en general es ascendente (Figura 5.A), aunque con cierta irregularidad en el período 2006-2011, lo que es posible se asocie a la evolución del tipo de cambio y el desempeño de los mercados de destino. A nivel regional, como ya se había evidenciado, el índice de O'Higgins obtiene un mejor desempeño en particular a partir del año 2006. Con menor desempeño sectorial se encuentra la región de Los Lagos, que registra un índice bajo en todo el período aunque creciente. En cambio, La Araucanía en el último período ha observado una ganancia relativa importante. No obstante, se aprecia que la brecha entre regiones se ha ampliado notablemente.

\section{Capital Humano}

Este indicador está compuesto por siete variables: cobertura en todos los niveles de educación: pre-básica, básica, media, pregrado y postgrado, puntaje medio en el SIMCE y promedio de años de escolaridad; su evolución anual se observa en la Figura 5.B.

Se obtiene un resultado con tendencia al alza en todas las regiones, aunque la posición relativa de la región de O'Higgins es dominante en la parte final del período. Por otro lado, se aprecia que Los Lagos tiene un bajo nivel en casi todo el ciclo, sin embargo, es la región que registra mayores variaciones, lo que se explica por el aumento que ha obtenido la cobertura en educación prebásica, básica y el porcentaje de matriculados en postgrado en el sector silvoagropecuario, variables que ingresan al indicador con alta ponderación (Tabla 1). El incremento de la cobertura en prebásica es más pronunciado en Los Lagos, de hecho aquí la matrícula en 2011 es 2,2 veces la del período inicial. Además, la matrícula en postgrados del sector solo se incrementa en La Araucanía y Los Lagos, siendo mayor el impacto en el último caso. No obstante, se evidencia una caída en la matrícula de carreras de pregrado en todas las regiones.

\section{Personas y Trabajo}

Este indicador está compuesto por variables: demográfica (tasa de natalidad), de empleo (ocupación en el sector y fuerza de trabajo), calidad de empleo (población cotizante en previsión de salud y ocupados dependientes capacitados) y bienestar social (pobreza, distribución de ingresos y denuncias por delitos). Se observa (Figura 5.C) un comportamiento estable tanto en el resultado final como en la posición relativa que ocupa cada región, con excepción de Los Lagos que pierde competitividad anual y notablemente desde el 2007. Las variables que más ponderan son el porcentaje de población con previsión de salud, esta ha aumentado significativamente en todas las regiones, no obstante en Los Lagos la cobertura es inferior, en la media es equivalente a la mitad de las otras regiones. A su vez, se obtiene que la pobreza se reduce en todo el período, y en términos porcentuales, esa caída es más importante en la región de O’Higgins.

No obstante, otras variables como la tasa de natalidad y la proporción de capacitados tiende a la baja en todas las regiones y las denuncias por delitos a aumentar, lo que se entiende como un indicador de inestabilidad.

\section{Infraestructura Comunitaria}

Este factor captura la cobertura de bienes básicos e incluye las variables distribución de agua, conexión a internet, establecimientos de educación y salud, con diez variables. Se observa que la región con mejor posición relativa es Los Lagos (Figura 5.D); al contrario, la con menor ubicación es O'Higgins. Aquí la variable que tiene mayor peso relativo es la disponibilidad de infraestructura en salud (servicios de atención primaria), establecimientos educacionales y 


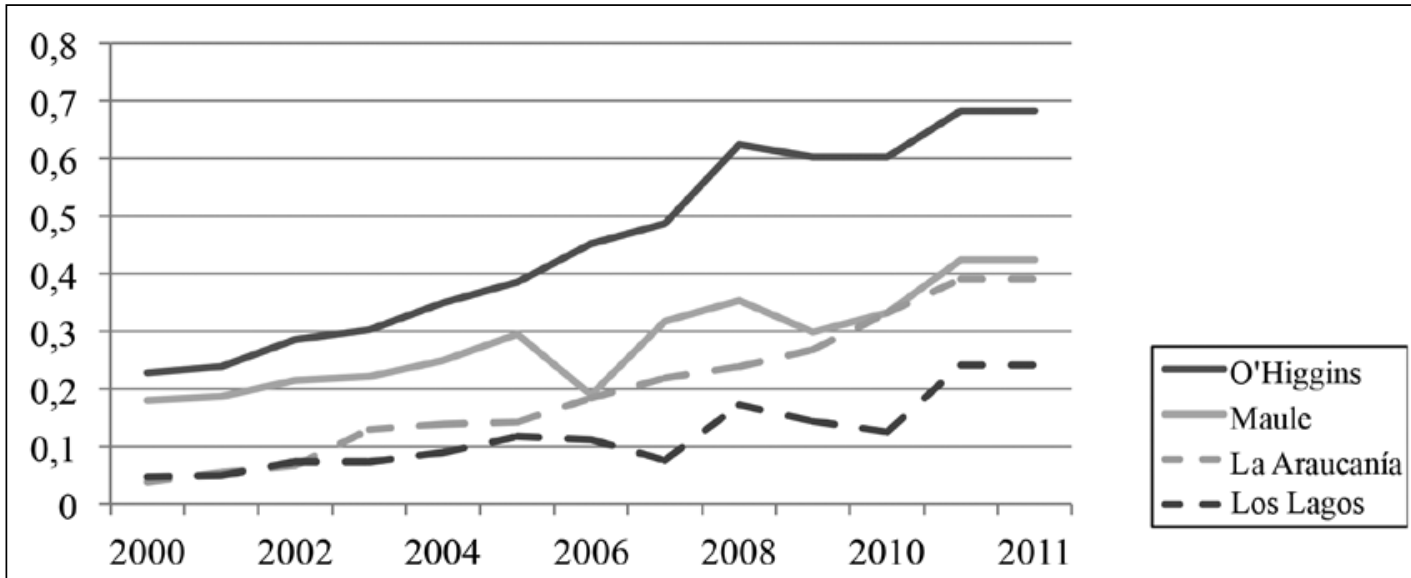

A. Factor Desempeño Económico por región y año

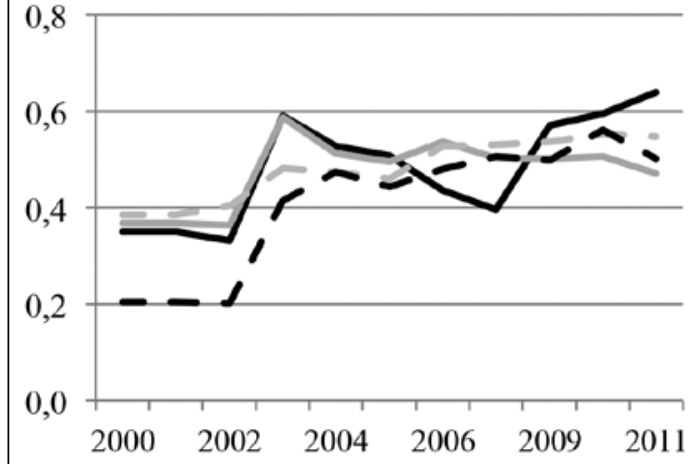

B. Capital humano

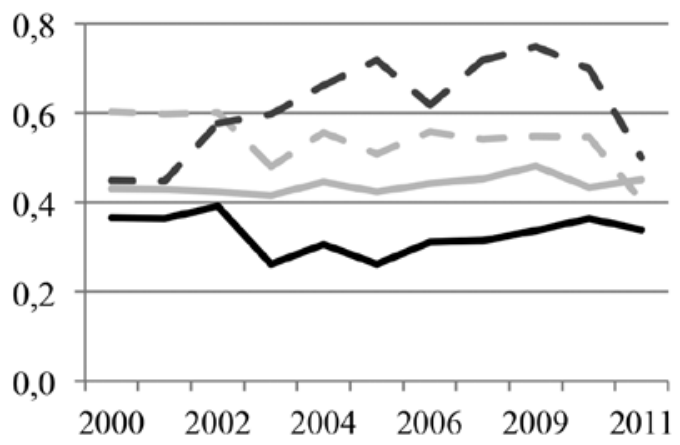

D. Infraestructura comunitaria
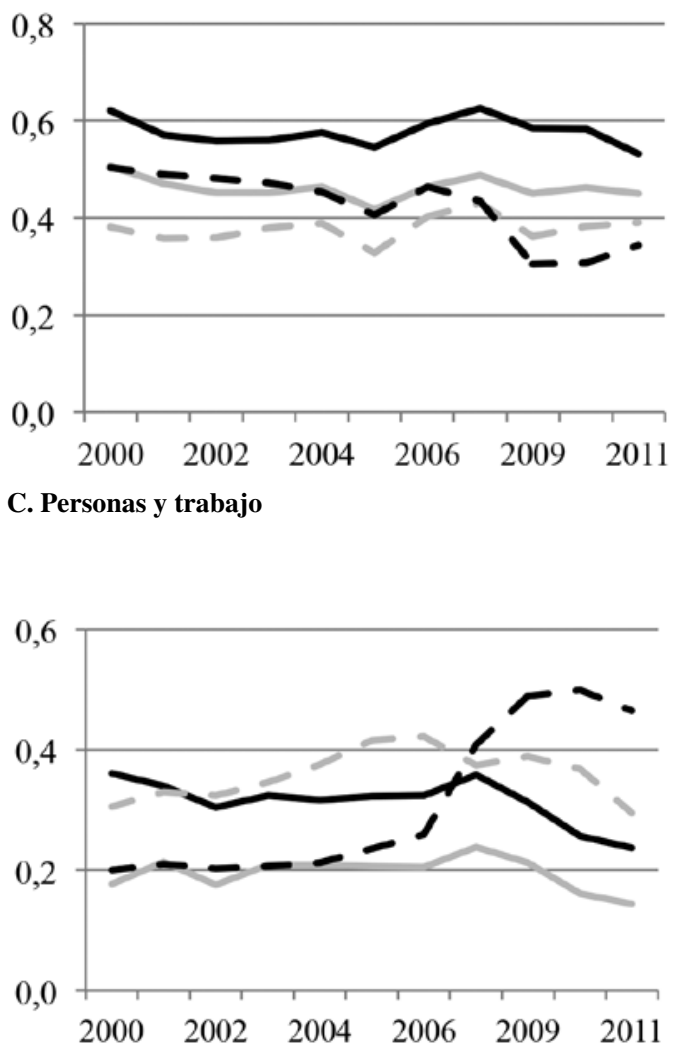

E. Rendimiento de recursos

Figura 5. Índice de competitividad parcial por factor, año y región.

Fuente: Elaboración propia. 
conexiones a internet, donde se obtiene que la disponibilidad en el promedio regional se ha duplicado, siendo superior en la infraestructura de salud para Los Lagos.

\section{Rendimiento de Recursos}

Este factor se compone de nueve variables, rendimientos de diversos cultivos (trigo, avena, cebada y papas), de animales (bovino, ovino, porcino), superficie silvícola y plantaciones de pino y eucalipto. El índice de La Araucanía, O'Higgins y Maule muestra una trayectoria estable desde 2003, aunque con una leve disminución en el último período explicado por la reducción de la actividad del rubro silvícola (Figura 5.E). A pesar de que ello también impacta a Los Lagos, esta logra revertir la posición desfavorable al principio del período, lo que se explica por la dinámica ascendente que tienen los rendimientos de cultivos en la región, pasando del último al primer lugar a partir del 2009, en particular por el rendimiento del cultivo de papas.

\section{Análisis impacto en el Desempeño Económico}

Si bien hasta ahora se ha estudiado la competitividad a partir de un indicador sintético, como lo indican los estudios a nivel mundial, en este caso resumiendo información de cinco factores tratados como independientes, también se sabe que los resultados económicos de un territorio están de alguna forma determinados por la dotación de factores no económicos. A su vez, en los resultados anteriores se observa que el desempeño económico es revelador del resultado final del indicador. Por lo que en este caso se va a indagar en la importancia que tienen los factores no económicos indicativos de competitividad en los resultados económicos. Para ello se estima una ecuación por variable del factor economía: producto interno bruto sectorial per cápita, la inversión mediante el FIA, las exportaciones agrícolas, pecuarias y forestales, considerando a cada una de ellas endógenas de los factores no económicos. En la Tabla 2 se observan los modelos estimados, en todos ellos se contrasta el test de normalidad de los residuos, solo en la estimación para FIA no se verifica.

Se obtiene que los factores con mayor poder explicativo son Personas y Trabajo y Capital Humano, este último deja de ser significativo solo para el caso de las exportaciones forestales. Por el contrario, los factores Infraestructura Comunitaria y Rendimiento de Recursos impactan significativamente solo en una de las variables que componen el factor Economía, el primero afecta al nivel de PIB sectorial y el segundo en las exportaciones pecuarias.

Finalmente, se plantea averiguar si el efecto de los factores en el desempeño económico es igual en todas las regiones, para ello se incorporan los territorios al modelo como variables ficticias,

Tabla 2. MCO, utilizando 48 observaciones, 4 unidades sección cruzada, 12 en serie temporal. Variable dependiente: PIB, XAGRI, XPECUA, XFOREST, FIA.

\begin{tabular}{|c|c|c|c|c|c|c|c|c|c|c|}
\hline & \multicolumn{2}{|c|}{ PIB } & \multicolumn{2}{|c|}{ XAGRI } & \multicolumn{2}{|c|}{ XPECUA } & \multicolumn{2}{|c|}{ XFOREST } & \multicolumn{2}{|c|}{ FIA } \\
\hline & Coef. & Est. t & Coef. & Est. t & Coef. & Est. t & Coef. & Est. t & Coef. & Est. t \\
\hline Constante & $-375,2 * *$ & $-2,2$ & $-1647,6^{*}$ & $-2,0$ & $-785,7$ & $-2,4 * *$ & $692,3^{* *}$ & 2,3 & 1301,8 & $2,6^{* *}$ \\
\hline $\mathrm{CH}$ & $540,2 * * *$ & 4,9 & $2133,1 * * *$ & 3,9 & 654,2 & $3,1 * * *$ & 99,6 & 0,5 & 772,8 & $2,3 * *$ \\
\hline PT & $1168,6^{* * *}$ & 6,1 & $3874,3^{* * *}$ & 4,0 & 1133,4 & $3,0 * * *$ & $-845,1 * *$ & $-2,4$ & $-1748,5$ & $-3,0 * * *$ \\
\hline $\mathrm{RR}$ & 99,0 & 0,8 & $-488,7$ & $-0,8$ & 518,9 & $2,2^{* *}$ & $-180,7$ & $-0,8$ & $-188,5$ & $-0,5$ \\
\hline IC & $-308,3 * *$ & $-2,4$ & $-986,6$ & $-1,5$ & $-166,1$ & $-0,67$ & $-363,6$ & $-1,5$ & $-561,9$ & $-1,4$ \\
\hline $\mathrm{R} 2$ & & 0,81 & & 0,68 & & 0,48 & & 0,15 & & 0,34 \\
\hline R2 corregido & & 0,79 & & 0,65 & & 0,43 & & 0,07 & & 0,28 \\
\hline $\mathrm{F}(4,43)$ & & 46,4 & & 23,0 & & 10,14 & & 1,91 & & 5,70 \\
\hline Valor p (de F) & & 0,00 & & 0,00 & & 0,00 & & 0,12 & & 0,00 \\
\hline \multicolumn{2}{|c|}{ Normalidad de residuos } & & & 1,46 & & 4,58 & & 24,69 & & 18,11 \\
\hline Chi-cuadrado & & 2,09 & & & & & & & & \\
\hline Valor p & & 0,35 & & 0,48 & & 0,10 & & 0,00 & & 0,00 \\
\hline
\end{tabular}

*Significativo al 10\%; **Significativo al 5\%; ***Significativo al $1 \%$. 
Tabla 3. MCO, utilizando 48 observaciones, 4 unidades sección cruzada, 12 en serie temporal. Variable dependiente: XAGRI, XPECUA. XFOREST y FIA, con Regiones

\begin{tabular}{|c|c|c|c|c|c|c|c|c|c|c|}
\hline & \multicolumn{2}{|c|}{ PIB } & \multicolumn{2}{|c|}{ XAGRI } & \multicolumn{2}{|c|}{ XPECUA } & \multicolumn{2}{|c|}{ XFOREST } & \multicolumn{2}{|c|}{ FIA } \\
\hline & Coef. & Est. t & Coef. & Est. t & Coef. & Est. t & Coef. & Est. t & Coef. & Est. $\mathrm{t}$ \\
\hline Constante & 129,4 & 0,8 & $-115,8$ & $-0,1$ & 60,2 & 0,1 & 40,01 & 0,10 & 503,0 & 0,80 \\
\hline $\mathrm{CH}$ & $347,7 * * *$ & 4,1 & $1364,6^{* * *}$ & 2,5 & $718,0 * * *$ & 3,3 & 192,71 & 0,93 & $924,9 * *$ & 2,74 \\
\hline PT & 48,4047 & 0,2 & 11,7 & 0,0 & $-0,2$ & $-0,0$ & 283,19 & 0,51 & $-411,6$ & $-0,45$ \\
\hline $\mathrm{RR}$ & $-135,684$ & $-0,9$ & $-1485,9^{*}$ & $-1,7$ & $-171,8$ & $-0,4$ & 163,90 & 0,49 & $-263,8$ & $-0,49$ \\
\hline IC & $-104,633$ & $-0,8$ & 144,3 & 0,1 & $-389,1$ & $-1,2$ & $-354,52$ & $-1,19$ & $-338,5$ & $-0,69$ \\
\hline ARAUCANÍA & $-52,2 * *$ & $-2,1$ & $-43,4$ & $-0,2$ & $-96,2$ & $-1,5$ & 91,51 & 1,57 & $233,1 * *$ & 2,45 \\
\hline MAULE & $74,36^{* *}$ & 2,4 & $359,6^{*}$ & 1,8 & $-163,9 * *$ & $-2,0$ & 19,52 & 0,26 & $-8,4$ & $-0,07$ \\
\hline O'HIGGINS & $266,4 * * *$ & 5,1 & $1100,7 * * *$ & 3,3 & 97,1 & 0,7 & $-180,48$ & $-1,42$ & $-117,7$ & $-0,57$ \\
\hline R2 & & 0,91 & & 0,76 & & 0,58 & & 0,28 & & 0,47 \\
\hline R2 corregido & & 0,90 & & 0,72 & & 0,51 & & 0,15 & & 0,29 \\
\hline $\mathrm{F}(4,43)$ & & 61,53 & & 18,8 & & 8,20 & & 2,26 & & 5,11 \\
\hline Valor p (de F) & & 0,00 & & 0,00 & & 0,00 & & 0,04 & & 0,00 \\
\hline \multicolumn{2}{|c|}{ Normalidad de residuos } & & & 1,44 & & 4,20 & & 20,89 & & \\
\hline Chi-cuadrado & & 0,293 & & & & & & & & 11,17 \\
\hline Valor p & & 0,864 & & 0,48 & & 0,12 & & 0,00 & & 0,00 \\
\hline
\end{tabular}

*Significativo al 10\%; **Significativo al 5\%; ***Significativo al $1 \%$.

dejando como referencia a la región de Los Lagos. Estos modelos presentan bajo nivel de significatividad, y en las exportaciones forestales en ninguna variable, además en este caso y la ecuación de FIA no se verifica normalidad de los residuos (Tabla 3). Sin embargo, de las otras estimaciones se obtiene que los impactos de los factores sobre el PIB sectorial son mayores en las regiones del Maule y O'Higgins y disminuye en la región de La Araucanía. A su vez el efecto sobre las exportaciones agrícolas solo es diferente en O'Higgins. En cambio, la región del Maule es significativamente mayor en el caso de las exportaciones pecuarias, y la región de La Araucanía solo genera impacto mayor en el caso de la Inversión de FIA.

\section{Conclusiones}

Este trabajo analiza el sector silvoagropecuario en cuanto a su competitividad y resultados económicos, desde una perspectiva regional. Así, los objetivos planteados son: (1) analizar la competitividad sectorial en las regiones que se especializan en el sector silvoagropecuario, y (2) evaluar el impacto de distintos factores no económicos de la competitividad sectorial en el desempeño económico del sector en las regiones.
Para lograr estos propósitos se entiende a la competitividad como un proceso localizado espacialmente, dinámico y de largo plazo. Luego, los factores indicativos de competitividad son: economía, capital humano, personas y trabajo, infraestructura comunitaria, rendimiento de recursos. Cada factor se mide a partir de un índice sintético anual, para ello se selecciona información anual de 40 variables, en el período 2000-2011.

Aun cuando los factores son al final indicadores agregados, ya que reúnen información de distintas variables en forma resumida, permiten acercarnos a la competitividad sectorial, de acuerdo con la definición asumida.

Del análisis global se obtiene que la región con mejor indicador de competitividad en forma sostenida es O'Higgins. Su mejor desempeño relativo se obtiene en los factores desempeño económico y personas y trabajo. La peor ubicación en el índice global la ocupa el Maule, pese a que es la segunda en desempeño económico, su bajo desempeño se ve confirmado por los factores rendimiento de recursos e infraestructura comunitaria en todo el período. Por otro lado, La Araucanía y Los Lagos obtienen resultados medios en el ranking global, a esta posición contribuyen los rendimientos de recursos y la dotación de infraestructura comunitaria, sin embargo están muy bajas en los factores desempeño económico y 
personas y trabajo. En Los Lagos es probable que se explique por la menor participación relativa del PIB del sector en la región, ya que en ella el sector pesca a aumentado en forma significativa su aporte superando al silvoagropecuario.

Se ha visto que el resultado del desempeño económico es decisivo en el valor del indicador global, y por lo tanto en la posición relativa que ocupan las regiones en cuanto a su competitividad sectorial. Sin embargo, la estimación del factor economía presenta una brecha importante entre las regiones que se ha ido ampliando en el tiempo, y el impacto de los factores capital humano y personas y trabajo es positivo y significativo tanto en el PIB sectorial como en las exportaciones agrícolas y pecuarias e inversión. A partir de lo anterior, planteamos la posibilidad de focalizar la intervención pública en factores no económicos específicos por región, de manera de generar un mejor impacto sobre el desempeño económico del sector en el territorio, por ejemplo una forma de avanzar en la región de Los Lagos es aumentando la cobertura en seguro de salud (FONASA e ISAPRE), lo que hoy es muy baja en relación con las otras regiones, además algunos elementos de la política se pueden abordar de forma conjunta, en particular entre las regiones que son cercanas en términos geográficos.
En la misma lógica una pregunta que nace de esta investigación es por qué los buenos resultados de O'Higgins no contagian al desempeño del Maule si son regiones adyacentes, ambas cercanas a la región Metropolitana. Si bien el Maule ocupa el segundo lugar en el factor economía en el período completo, se observa una pérdida importante en los últimos años (Figura 5.A), desde 2010 similar al resultado de La Araucanía.

No obstante, es probable que sea necesario plantear ciertas políticas de forma diferenciada, en particular en variables asociadas a infraestructura comunitaria y rendimiento de recursos. Como se observó, al incorporar a las regiones en los modelos se obtuvo que los impactos sobre el PIB sectorial son mayores en las regiones del Maule y O'Higgins, lo que debiese ser aprovechado en el primer caso. A su vez el efecto sobre las exportaciones agrícolas solo es diferente en O'Higgins, lo que podría atraer a las actividades maulinas.

Por último, el análisis realizado incluye a sectores agregados, lo que no permite evaluar la diversidad del sector en cada región, el desafío ahora es profundizar en la competitividad de los subsectores y los determinantes de su desempeño económico a niveles inferiores de agregación sectorial y territorial.

\section{Literatura Citada}

Araoz, M.

1997. La integración como instrumento para incrementar la competitividad en un mundo globalizado: perspectivas en la Comunidad Andina. La Integración Regional y los Desafíos de la Competitividad y la Convergencia: Requisitos, Estrategias y Perspectivas, CEFIR, 1998. Montevideo, Uruguay, pp. 15-24.

Cheshire, P.C.; Hay, D.

1986. The development of the European urban system, 1971-1981. In: Ewers, H.J.; Goddard, J.B.; Matzerath, H. (eds.): The future of the metropolis. Berlin - London Paris - New York. Economic Aspects. Berlin, New York, pp. 149-169.

Comisión Europea

2003. A study on the factors of regional competitiveness. A draft final report for The European Commission DirectorateGeneral Regional Policy, Bruselas, Cambridge Econometrics Ecorys-Nei, University of Cambridge. http://ec.europa. eu/regional_policy/sources/docgener/studies/pdf/3cr/ competitiveness.pdf. Consultado: 20/Jun/2014.

Cuadrado, R.

1992. Cuatro décadas de economía del crecimiento regional en Europa: principales corrientes doctrinales.
En: Economía española, cultura y sociedad: homenaje a Juan Velarde Fuertes ofrecido por la Universidad Complutense. García J. y Serrano J. (Coord.), 2: pp. 525-562.

Gobierno Regional Los Lagos

2009. Estrategia regional desarrollo Los Lagos 2009-

2020. http://www.goreloslagos.cl/gobierno_regional/ estrategia_regional_desarrollo.html. Consultado: 20/ Jun/2014.

Hatzichronoglou, T.

1996. Globalisation and Competitiveness: Relevant Indicators, OECD Science, Technology and Industry Working Papers, 1996/05, OECD Publishing. http://dx.doi. org/10.1787/885511061376

IMD World Competitiveness Center (WCC)

2014. Methodology and Principles of Analysis. http:// www.imd.org/uupload/imd.website/wcc/methodology. pdf. Consultado: 5/Jul/ 2014.

Kresl, P.; Balwant S.

1996. Competitiveness and the urban economy: twenty-four large US metropolitan areas. Urban Studies, $36(5 / 6)$ : 1017-1027. 
Lever, W.F.; Turok I.

1999. Competitive Cities: Introduction to the Review. Urban Studies, 36 (5/6): 791-794.

Marcovitch, J.

1994. Competitiveness in Brasil. Revista Espacios, 15 (1). http://www.revistaespacios.com/a94v15n01/21941501. html. Consultado: 20/Jun/2014.
Porter, M.

1995. The competitive advantage of the inner city. Harvard Business Review, 73 (3): 55-71.

Schwab K.

2011.The Global Competitiveness Report 2010-2011, World Economic Forum. Geneva. Disponible en: http://www.bpcc.pt/ files/WEF_GCR_Portugal_2010-11.pdf.Consultado:20/Jun/2014. 